\title{
Endovascular placement of iodine-125 seed strand and stent combined with chemoembolization for treatment of hepatocellular carcinoma with portal vein tumor thrombus
}

\author{
Suming Zhao, Xiaohu Yang, Weiwei Gu, Zhuxin Gu, Hui Zhao \\ Department of Interventional Radiology, Affiliated Hospital of Nantong University, Nantong 226001, China \\ Correspondence to: Hui Zhao. Department of Interventional Radiology, Affiliated Hospital of Nantong University, Nantong 226001, China. \\ Email: zhaohui800@163.com.
}

\begin{abstract}
A 59-year-old man was admitted to the hospital because of fatigue and marasmus for over 1 month. Computed tomography (CT) examination implied huge primary hepatocellular carcinoma (PHC) with tumor thrombus in the main portal vein (MPV). It also observed the mild expansion of intrahepatic bile ducts in the left lobe of the liver and lymph node metastasis to retroperitoneum, clearance between liver and stomach and porta hepatis. This video was conducted to show portal vein stenting (PVS) and transcatheter arterial chemoembolization (TACE) combined with endovascular implantation of iodine-125 $\left({ }^{125} \mathrm{I}\right)$ seeds for treating the patient with Hepatocellular carcinoma (HCC) with portal vein tumor thrombus (PVTT).
\end{abstract}

Keywords: Portal vein stenting (PVS); iodine-125 seed strand $\left({ }^{125} \mathrm{I}\right.$ seed strand); transcatheter arterial chemoembolization (TACE)

Submitted Jul 10, 2018. Accepted for publication Jul 24, 2018.

doi: $10.21037 /$ tcr.2018.07.23

View this article at: http://dx.doi.org/10.21037/tcr.2018.07.23

\section{Introduction}

Hepatocellular carcinoma (HCC) is one of the most common malignant tumors worldwide. Portal venous invasion is reported in $12.5-39.7 \%$ of patients with advanced HCC (1). When the main portal vein (MPV) was involved by tumor thrombus, the patient's prognosis was extremely poor (2). For these patients, there is no effective treatment and the optimal treatment remains controversial. Most HCCs with portal vein tumor thrombus (PVTT) are technically unresectable and they are not suitable for curative therapies. As a palliative treatment, transcatheter arterial chemoembolization (TACE) treatment for HCC with PVTT is safe and effective when there is sufficient collateral circulation. However, PVTT limits the effect of TACE on HCC and has a strong negative impact on the therapeutic effect (3). Therefore, opening the occlusion of the portal vein caused by PVTT and reperfusing the portal vein could improve the success of TACE, and this could be achieved using portal vein stenting (PVS). PVS combined with TACE has been successfully used in the treatment of HCC with PVTT. However, PVS effectively removes the portal vein obstruction but do not treat the thrombus per se, and stent restenosis may happen. Radioactive seed implantation is used in a variety of solid tumors. Iodine- 125 $\left({ }^{125} \mathrm{I}\right)$ seed implantation was attempted to treat HCC with PVTT and achieved excellent therapeutic efficacy (4) with a good safety profile (Figure 1).

\section{Operative techniques}

(I) Perform percutaneous transhepatic portal $6 \mathrm{~F}$ vagina vasorum implantation and CTAP by $5 \mathrm{~F}$ pigtail catheter. It shows that the filling defect at main portal vein in the size of approximately $8 \mathrm{~mm} \times 5 \mathrm{~cm}$;

(II) Remove pigtail catheter, bring in the super smooth guide wire and small wire adopted in percutaneous transhepatic cholangiography drainage), remove vasorum and then place it by super smooth guide wire. By replacement of the vasorum, long super smooth guide wire is inside the vasorum while the short one outside; 


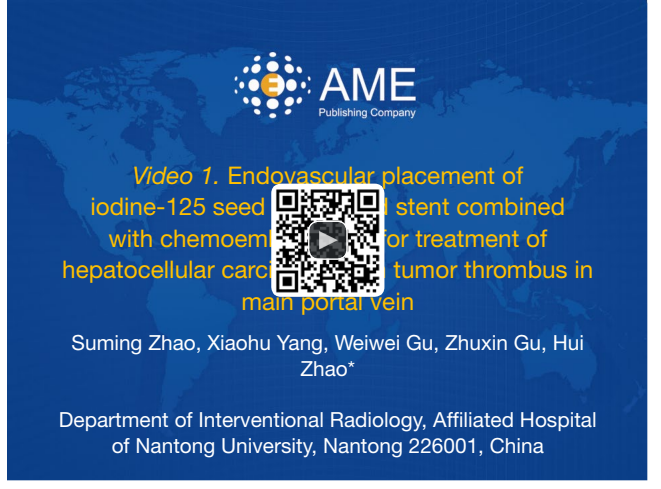

Figure 1 Endovascular placement of iodine-125 seed strand and stent combined with chemoembolization for treatment of hepatocellular carcinoma with tumor thrombus in main portal vein (5).

Available online: http://www.asvide.com/article/view/26844

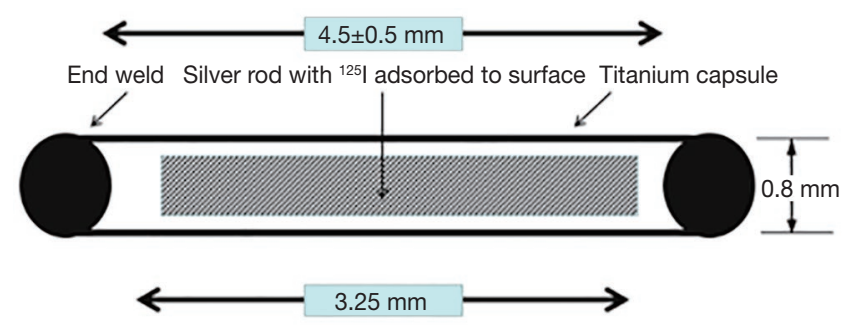

Figure $2{ }^{125} \mathrm{I}$ seed. Radioactivity of each Iodine-125 seed is 25.9 $\mathrm{MBq}$ with a half-life of 59.4 days.

(III) Preparation of seed strand: radioactivity of each ${ }^{125} \mathrm{I}$ seed is $25.9 \mathrm{MBq}$ with a half-life of 59.4 days. The number of ${ }^{125}$ I seeds planned to be implanted was calculated by the following formula: length of obstructed MPV (mm)/4.5+2 (Figure 2);

(IV) Release ev3 stent with the size of $14 \mathrm{~mm} \times 8 \mathrm{~cm}$ by smooth guide wire, guide it into the vagina vasorum of PTCD by small wires, remove small wires, and then push prepared ${ }^{125}$ I seed strand;

(V) Angiography shows that portal vein is smooth with stent in the right place and ${ }^{125} \mathrm{I}$ seed strand is in a good condition;

(VI) When the sheath is slowly withdrawing to liver parenchyma, use four coiling in the size of $3 \mathrm{~mm} \times 3 \mathrm{~cm}$ to seal the puncture path;

(VII) Perform percutaneous puncture from right femoral artery to sheath, and then guide $5 \mathrm{~F} \mathrm{RH}$ tube to super selective hepatic artery. Angiography shows the staining of giant tumor in right lobe of liver, which is significant in three feeding arteries. Prescribe such drugs for embolization as Pirarubicin $30 \mathrm{mg}$ + Oxaliplatin $50 \mathrm{mg}$ + Iodinated Oil $25 \mathrm{~mL}$ + PVA (500-700 $\mu \mathrm{m})$.

\section{Comments}

(I) TACE cannot be directly performed on the patients with primary hepatocellular carcinoma (PHC) and PVTT. The combination of portal vein stent and endovascular implantation of ${ }^{125} \mathrm{I}$ seed strand can open the portal vein and conduct radiation therapy on PVTT by radioactive seeds strand;

(II) After opening the portal vein, TACE can be performed and the focus in portal vein or liver can be treated;

(III) Due to the huge tumor, complete embolization at one time may cause damage on liver function and severe postoperative reactions, so fractionated embolization should be adopted.

\section{Acknowledgments}

Funding: None.

\section{Footnote}

Conflicts of Interest: All authors have completed the ICMJE uniform disclosure form (available at http://dx.doi. org/10.21037/tcr.2018.07.23). The authors have no conflicts of interest to declare.

Ethical Statement: The authors are accountable for all aspects of the work in ensuring that questions related to the accuracy or integrity of any part of the work are appropriately investigated and resolved. All procedures performed in study involving human participants were in accordance with the ethical standards of the institutional and/or national research committee(s) and with the Declaration of Helsinki (as revised in 2013). Written informed consent was obtained from the patient for publication of this manuscript and any accompanying images.

Open Access Statement: This is an Open Access article distributed in accordance with the Creative Commons Attribution-NonCommercial-NoDerivs 4.0 International 
License (CC BY-NC-ND 4.0), which permits the noncommercial replication and distribution of the article with the strict proviso that no changes or edits are made and the original work is properly cited (including links to both the formal publication through the relevant DOI and the license). See: https://creativecommons.org/licenses/by-nc-nd/4.0/.

\section{References}

1. Shi J, Lai EC, Li N, et al. A new classification for hepatocellular carcinoma with portal vein tumor thrombus. J Hepatobiliary Pancreat Sci 2011;18:74-80.

2. Zhou L, Rui JA, Wang SB, et al. Risk factors of poor prognosis and portal vein tumor thrombosis after curative resection of solitary hepatocellular carcinoma. Hepatobiliary Pancreat Dis Int 2013;12:68-73.

3. Sun JH, Zhou T, Zhu T, et al. Portal Vein Stenting

Cite this article as: Zhao S, Yang X, Gu W, Gu Z, Zhao H. Endovascular placement of iodine-125 seed strand and stent combined with chemoembolization for treatment of hepatocellular carcinoma with portal vein tumor thrombus. Transl Cancer Res 2018;7(4):1161-1163. doi: 10.21037/ tcr.2018.07.23
Combined with Iodine-125 Seeds Endovascular Implantation Followed by Transcatheter Arterial Chemoembolization for Treatment of Hepatocellular Carcinoma Patients with Portal Vein Tumor Thrombus. Biomed Res Int 2016;2016:3048261.

4. Yang M, Fang Z, Yan Z, et al. Transarterial chemoembolization (TACE) combined with endovascular implantation of an iodine-125 seed strand for the treatment of hepatocellular carcinoma with portal vein tumour thrombosis versus TACE alone: a two-arm, randomised clinical trial. J Cancer Res Clin Oncol 2014;140:211-9.

5. Zhao S, Yang X, Gu W, et al. Endovascular placement of iodine-125 seed strand and stent combined with chemoembolization for treatment of hepatocellular carcinoma with tumor thrombus in main portal vein. Asvide 2018;5:727. Available online: http://www.asvide.com/article/view/26844 